\title{
Quantum State Transfer Between Matter and Light
}

\author{
D. N. Matsukevich, A. Kuzmich * \\ School of Physics, Georgia Institute of Technology, \\ Atlanta, GA 30332, USA \\ *To whom correspondence should be addressed; E-mail: alex.kuzmich@physics.gatech.edu.
}

\begin{abstract}
We report on the coherent quantum state transfer from a two-level atomic system to a single photon. Entanglement between a single photon (signal) and a two-component ensemble of cold rubidium atoms is used to project the quantum memory element (the atomic ensemble) onto any desired state by measuring the signal in a suitable basis. The atomic qubit is read out by stimulating directional emission of a single photon (idler) from the (entangled) collective state of the ensemble. Faithful atomic memory preparation and read-out are verified by the observed correlations between the signal and the idler photons. These results enable implementation of distributed quantum networking.
\end{abstract}

\section{Introduction}

The ability to coherently transfer quantum information between photonic- and material-based quantum systems is a prerequisite for all practical distributed quantum computation and scalable quantum communication protocols (1). The importance of this process is rooted in the fact that matter-based quantum systems provide excellent long-term quantum memory storage, whereas long-distance communication of quantum information will most certainly be accomplished by 
coherent propagation of light, often in the form of single photon pulses.

In the microwave domain, coherent quantum control has been obtained with single Rydberg atoms and single photons (2); significant advances have also been made in ion trapping information processing (3-5). Particularly, an entangled state of an ion and a photon has been produced (6); however, to convert a single ion (atom) qubit state into a photonic state, strong coupling to a single cavity mode is required. Trapped atoms or ions localized inside of highfinesse cavities offer a natural paradigm for coherent, reversible matter-light interactions $(7,8)$, although technical challenges make these systems difficult to realize in practice.

Optically thick atomic ensembles have emerged recently as an alternative for the light-matter interface $(9,10)$. Duan, Lukin, Cirac and Zoller (DLCZ) (11) have made a theoretical proposal aimed at long-distance quantum communication that utilizes the quantum memory capability of atomic ensembles. Important initial steps towards realization of the DLCZ protocol have been made in which non-classical radiation has been produced from an atomic ensemble, thereby demonstrating the collective enhancement (12-15).

Here we report on the experimental realization of coherent quantum state transfer from a matter qubit onto a photonic qubit, utilizing an optically thick cold atomic cloud. Our experiment involves three steps: 1) an entangled state between a single photon (signal) and a single collective excitation distributed over many atoms in two distinct optically thick atomic samples is generated. 2) measurement of the signal photon projects the atomic ensembles into a desired state, conditioned on the choice of the basis and the outcome of the measurement. This atomic state is a nearly maximally entangled state between two distinct atomic ensembles. 3) this nearly maximally entangled atomic state is converted into a single photon (idler) emitted into a well-defined mode, without using a high-finesse cavity. These three ingredients constitute a complete set of tools required to build an arbitrary large-scale quantum network (11).

As illustrated in Fig.1A, the classical laser pulses used in the generation and verification 
procedures define the two distinct pencil-shape components of the atomic ensemble that form our memory qubit, L and R. Fig.1B indicates schematically the structure of the four atomic levels involved, $|a\rangle,|b\rangle,|c\rangle$ and $|d\rangle$. The experimental sequence starts with all of the atoms prepared in state $|a\rangle$. A write pulse tuned to the $|a\rangle \rightarrow|c\rangle$ transition is split into two beams by a polarizing beam splitter (PBS1) and passed through the atomic sample. The light induces spontaneous Raman scattering on the $|c\rangle \rightarrow|b\rangle$ transition. The classical write pulse is so weak that less than one photon is scattered in this manner into the forward direction mode for each pulse in either L or R. The forward scattered mode is dominantly correlated with a distinct collective atomic state (11). In the first order of perturbation theory in the atom-light coupling $\chi$, the atom-light state is

$$
|\Psi\rangle \sim|a\rangle_{1} \ldots|a\rangle_{N_{L}+N_{R}}\left|0_{p}\right\rangle_{L}\left|0_{p}\right\rangle_{R}+\chi\left(\left|L_{a}\right\rangle\left|1_{p}\right\rangle_{L}\left|0_{p}\right\rangle_{R}+\left|R_{a}\right\rangle\left|0_{p}\right\rangle_{L}\left|1_{p}\right\rangle_{R}\right)
$$

We have defined two effective states of the atomic ensembles:

$$
\begin{array}{r}
\left|L_{a}\right\rangle=\sum_{i=1}^{N_{L}} g_{i}|a\rangle_{1} \ldots|b\rangle_{i} \ldots|a\rangle_{N_{L}} \ldots|a\rangle_{N_{L}+N_{R}} \\
\left|R_{a}\right\rangle=\sum_{j=N_{L}+1}^{N_{L}+N_{R}} g_{j}|a\rangle_{1} \ldots|a\rangle_{N_{L}} \ldots|b\rangle_{j} \ldots|a\rangle_{N_{L}+N_{R}},
\end{array}
$$

with the weights $g_{i}, g_{j}$ determined by the write field intensity distribution, $\sum_{i=1}^{N_{L}}\left|g_{i}\right|^{2}=1$, $\sum_{j=N_{L}+1}^{N_{L}+N_{R}}\left|g_{j}\right|^{2}=1(16,17) . \quad\left|L_{a}\right\rangle$ and $\left|R_{a}\right\rangle$ have properties of a two-level system (qubit): $\left\langle L_{a} \mid L_{a}\right\rangle=1,\left\langle R_{a} \mid R_{a}\right\rangle=1,\left\langle L_{a} \mid R_{a}\right\rangle=0$. Although the interaction of the light with the atoms is non-symmetric with respect to permutation of atoms, the second term in Eq11 in fact describes a strongly entangled atom-photon state in the sense of (17). Using PBS4 and a half wave plate inserted into one of the channels, we map the two spatial modes associated with the two ensembles into a single spatial mode with polarization encoding of the light's origin: $\left|1_{p}\right\rangle_{L} \rightarrow|H\rangle_{s} ;\left|1_{p}\right\rangle_{R} \rightarrow|V\rangle_{s}$, where $H$ and $V$ indicate horizontal and vertical polarization, respectively, and $s$ denotes signal. Next, the light is passed through an arbitrary polarization 
state transformer $R_{s}\left(\theta_{s}, \phi_{s}\right)$ and a polarizer PBS5, so that the state at the output of PBS5 is

$$
\left|H^{\prime}\right\rangle=\cos \left(\theta_{s}\right) e^{i \phi_{s}}|H\rangle_{s}+\sin \left(\theta_{s}\right)|V\rangle_{s}
$$

and is directed onto a single-photon detector D1. When D1 detects a photon, the joint state in Eq. 1 is projected into the desired atomic state

$$
\left|\Psi_{a}\right\rangle=\cos \left(\theta_{s}\right) e^{-i \phi_{s}}\left|L_{a}\right\rangle+\sin \left(\theta_{s}\right) e^{i \eta_{s}}\left|R_{a}\right\rangle
$$

which is an entangled state of the two atomic samples $L$ and $R$. Phase $\eta_{s}$ is determined by the difference in length of the two paths $L$ and $R$. After a variable delay time $\Delta t$ we convert the atomic excitation into a single photon by illuminating the atomic ensemble with a pulse of light near resonant with the $|b\rangle \rightarrow|d\rangle$ transition. For an optically thick atomic sample, the photon will be emitted with high probability into the spatial mode determined by the write pulse $(11,16)$, achieving memory read-out:

$$
\left|\Psi_{a}\right\rangle=\cos \left(\theta_{s}\right) e^{-i \phi_{s}}\left|L_{a}\right\rangle+\sin \left(\theta_{s}\right) e^{i \eta_{s}}\left|R_{a}\right\rangle \rightarrow|\Psi\rangle_{i}=\cos \left(\theta_{s}\right) e^{-i \phi_{s}}|H\rangle_{i}+\sin \left(\theta_{s}\right) e^{i\left(\eta_{i}+\eta_{s}\right)}|V\rangle_{i} .
$$

That is, the polarization state of the idler photon $i$ is uniquely determined by the observed state of the signal photon. Alternatively, one could store the signal in a fiber until after the read-out. In that case, the two-photon signal-idler state would be a maximally entangled state:

$$
\left|\Psi_{M}\right\rangle=\frac{1}{\sqrt{2}}\left(|H\rangle_{s}|H\rangle_{i}+e^{i\left(\eta_{s}+\eta_{i}\right)}|V\rangle_{s}|V\rangle_{i}\right)
$$

A magneto-optical trap (MOT) of ${ }^{85} \mathrm{Rb}$ is used to provide an optically thick atomic cloud for our experiment (Fig.1). The ground states $\{|a\rangle ;|b\rangle\}$ correspond to the $5 S_{1 / 2}, F=\{3,2\}$ levels of ${ }^{85} \mathrm{Rb}$, while the excited states $\{|c\rangle ;|d\rangle\}$ represent the $\left\{5 P_{3 / 2}, F=3 ; 5 P_{1 / 2}, F=2\right\}$ levels of the $\left\{D_{2}, D_{1}\right\}$ lines at $\{780 ; 795\} \mathrm{nm}$, respectively. The experimental sequence starts with all of the atoms prepared in state $|a\rangle$ via optical pumping, after shutting off the trapping and cooling light. 
A 140 ns long write pulse tuned to the $|a\rangle \rightarrow|c\rangle$ transition is split into two beams by a polarizing beamsplitter PBS1 and focused into two regions of the MOT about $1 \mathrm{~mm}$ apart with Gaussian waists of about $50 \mu \mathrm{m}$. PBS2 and PBS3 separate the horizontally polarized component of the forward scattered light from the vertically polarized classical pulse. After being mixed by PBS4, the light goes through the quarter- and the half-wave plates that provide the state transformation $R_{s}\left(\theta_{s}, \phi_{s}\right)$. The light continues to another polarizer PBS5, and is directed to a single photon detector D1. Detection of one photon by D1 prepares the atomic ensemble in any desired state in the basis of $\left|L_{a}\right\rangle,\left|R_{a}\right\rangle$ determined by $R_{s}\left(\theta_{s}, \phi_{s}\right)$ and thereby concludes the preparation of the quantum memory qubit.

Following memory state preparation, the read-out stage is performed. After a user-programmable delay $\Delta t$, a $115 \mathrm{~ns}$ long read pulse tuned to the $|b\rangle \rightarrow|d\rangle$ transition illuminates the two atomic ensembles. This accomplishes a transfer of the memory state onto the single photon (idler) emitted by the $|d\rangle \rightarrow|a\rangle$ transition. After passing through the state transformer $R_{i}\left(\theta_{i}, \phi_{i}\right)$ and PBS6, the two polarization components are directed onto single-photon detectors (D2, D3) thus accomplishing measurement of the idler photon, and hence the memory qubit, in a controllable arbitrary basis.

As in any real experiment, various imperfections prevent the read-out of the quantum memory (idler photon) from being identical to the state that we intended to write into the memory. To quantify the degree to which we faithfully prepare and read-out the quantum memory, we measure the polarization correlations between the signal and idler photons. The observed correlations allow us to characterize the extent to which our procedures are working. To investigate the storage capabilities of our memory qubit quantitatively, we use time-resolved detection of the signal and idler photons for two values of delay $\Delta t$ between the application of the write and read pulses, $100 \mathrm{~ns}$ and $200 \mathrm{~ns}$. The electronic pulses from the detectors are gated with $250 \mathrm{~ns}$ and $140 \mathrm{~ns}$ windows centered on the time determined by the write and read light pulses, 
respectively. Afterwards, the electronic pulses are fed into a time-interval analyzer (with $\delta=2$ ns time resolution). In order to measure the correlation between the photons produced by the write and read pulses, the output of D1 is fed into the "Start" input of a time-interval analyzer, and the outputs of D2 and D3 are fed into two "Stop" inputs. A coincidence window imposed by the data acquisition software selects a time interval between the arrival of the idler and signal of $(0,80) \mathrm{ns}$ for $\Delta t=100 \mathrm{~ns}$ and $(25,145) \mathrm{ns}$ for $\Delta t=200 \mathrm{~ns}$.

We first measure the conditional probabilities of detecting a certain state of the idler (hence, of the quantum memory state) in the basis of $|H\rangle_{i}$ and $|V\rangle_{i}$, given the observed state of the signal photon. Varying the angle $\theta_{s}$ produces the correlation patterns shown in Fig.2A for $\Delta t=100 \mathrm{~ns}$. Conditional probabilities at the point of maximum correlation are shown in Fig.2B and the first line of Table 1. To verify faithful memory preparation and read-out, we repeat the correlation measurement in a different basis, of states $\left(|H\rangle_{i} \pm|V\rangle_{i}\right) / \sqrt{2}$, by choosing the $\theta_{i}=45$ degrees, $\phi_{i}=0$ degrees, and $\phi_{s}=-\left(\eta_{s}+\eta_{i}\right)$ in the state transformers $R_{s}$ and $R_{i}$. We vary $\theta_{s}$, with the measured interference fringes displayed in Fig. 3A. Table 1 (second line) and Fig. 3B show the conditional probabilities at the point of maximum correlations. These probabilities are different from $1 / 2$ only when the phase coherence between the two states of the atomic qubit is preserved in the matter-to-light quantum state mapping.

From these measured correlations, we determine the fidelity of the reconstruction of our intended quantum memory state $\left|\Psi_{I}\right\rangle$ in the idler, $\left|\left\langle\Psi_{I} \mid \Psi_{i}\right\rangle\right|^{2}$. The fidelity is given by the value of the corresponding conditional probability at the point of maximum correlation, presented in Table 1 (we choose the lower of the two values as the lower bound). For states in the $\theta_{i}=0$ degree basis, we find $F_{0}=0.88 \pm 0.03$, clearly exceeding the classical boundary of $2 / 3(18)$. For the $\theta_{i}=45$ degree basis, we found $F_{45}=0.75 \pm 0.02$, again significantly violating the classical limit. These fidelities give a lower bound for both the fidelities of the memory preparation and the read-out steps, which we do not measure separately. 
Another way to quantify the performance of our quantum state transfer is to calculate the fidelity of entanglement between the signal and idler photons $F_{s i}$. The lower bound on $F_{s i}$ is given by the overlap of the measured density matrix with the maximally entangled state we seek to achieve $\left|\Psi_{M}\right\rangle$ given by Eq[5 $F_{s i}=\left\langle\Psi_{M}\left|\rho_{s i}\right| \Psi_{M}\right\rangle$ (19). We calculated $F_{s i}=0.67 \pm 0.02$, substantially greater that the classical limit of $1 / 2(6,19)$.

At a longer delay of $200 \mathrm{~ns}$ the fidelities in the $\theta_{i}=0$ degrees and $\theta_{i}=45$ degrees bases are $F_{0}=0.79 \pm 0.04$ and $F_{45}=0.74 \pm 0.04$, while fidelity of entanglement is $F_{s i}=0.63 \pm 0.03$. For both values of $\Delta t$, we analyze the fidelity of entanglement as a function of the delay between the detections of the signal and the idler. We split the full coincidence window into four equal intervals, and calculated entanglement of formation for each one (Fig.4). From these results, we conclude that our quantum memory has a useful operational time of about $150 \mathrm{~ns}$. The lifetime of coherence between the levels $|a\rangle$ and $|b\rangle$ determines the lifetime of the quantum memory and is limited by the magnetic field of the trapping quadrupole field of the MOT (12).

Non-zero coincidence counts in the minima of Fig. 2A are due to transmission losses and non-ideal spatial correlations between the signal and idler photons. The residual interferometric drifts in $\eta_{s}+\eta_{i}$ further reduce the visibility of Fig. 3A compared to Fig. 2A, resulting in a degradation of the fidelities. Losses also reduce the rate of entanglement generation. The rate of signal photon detections (and hence, atomic qubit preparation) is given by $R_{s}=\alpha n_{s} R \simeq$ $300 s^{-1}$, where $\alpha=0.05$ is the measured transmission efficiency for the write beam (which includes 0.60 detection efficiency), and $R=4.7 \times 10^{5} s^{-1}$ is the repetition rate of the experiment. Therefore, the inferred average photon number in the forward scattered mode per pulse is $n_{s} \simeq$ $1.4 \times 10^{-2}$. The coincident signal-idler detection rate is $R_{s i}=\zeta R_{s}=\zeta \alpha n_{s} R \simeq 0.4 s^{-1}$, where $\zeta \equiv \beta \xi \simeq 1.1 \times 10^{-3}$. The measured transmission and detection efficiency for the read beam is $\beta \simeq 0.04$, so we infer the efficiency of quantum state transfer from the atoms onto the photon $\xi \simeq 0.03$. 
We have realized a quantum node by combining the entanglement of an atomic and photonic qubits with the atom-photon quantum state transfer. By implementing the second node at a different location, and performing a joint detection of the signal photons from the two nodes, the quantum repeater protocol (11), as well as distant teleportation of an atomic qubit may be realized. Based on this work, we estimate the rate for these protocols to be $R_{2} \simeq\left(\beta \xi \alpha n_{s}\right)^{2} R \simeq$ $3 \times 10^{-7} s^{-1}$. However, improvements in $\xi$ that are based on increasing the optical thickness of atomic samples (16), as well as elimination of transmission losses could provide several orders of magnitude increase in $R_{2}$. Our results also demonstrate the possibility of realizing quantum nodes consisting of multiple atomic qubits by using multiple beams of light. This approach shows promise for implementation of distributed quantum computation $(20,21)$.

\section{References and Notes}

1. I. Chuang, M. Nielsen, Quantum computation and quantum information, (Cambridge University Press, 2000).

2. S. Haroche, J. M. Raimond, M. Brune, in Experimental Quantum Computation and Information, eds. F. de Martini and C. Monroe), 37-66 (Proc. Int. School of Physics Enrico Fermi, course CXLVIII, IOS Press, Amsterdam, 2002).

3. C. A. Sackett et al., Nature 404, 256 (2000).

4. M. D. Barrett et al, Nature 429, 737 (2004).

5. M. Riebe et al, Nature 429, 734 (2004).

6. B. B. Blinov, D. L. Moehring, L.-M. Duan, C. Monroe, Nature 428, 153 (2004).

7. S. Bose, P. L. Knight, M. B. Plenio, V. Vedral, Phys. Rev. Lett. 83, 5158 (1999). 
8. H. J. Kimble, Phys. Scr. 76, 127 (1998).

9. A. Kuzmich, E. S. Polzik, in Quantum information with continuous variables, (eds. S. L. Braunstein and A. K. Pati, Kluwer, 2003).

10. M. D. Lukin, Rev. Mod. Phys. 75, 457 (2003).

11. L.-M. Duan, M. D. Lukin, I. J. Cirac, P. Zoller, Nature 414, 413 (2001).

12. A. Kuzmich et al., Nature 423, 731 (2003).

13. C. H. van der Wal et al., Science 301, 196 (2003).

14. W. Jiang, C. Han, P. Xue, L.-M. Duan, G. C. Guo, Phys. Rev. A 69, 043819 (2004).

15. C. W. Chou, S. V. Polyakov, A. Kuzmich, H. J. Kimble, Phys. Rev. Lett. 92, 213601 (2004).

16. L.-M. Duan, J. I. Cirac, P. Zoller, Phys. Rev. A 66, 023818 (2002).

17. A. Kuzmich, T. A. B. Kennedy, Phys. Rev. Lett. 92, 030407 (2004).

18. M. Horodecki, P. Horodecki, R. Horodecki, Phys. Rev. A 60, 1888 (1994).

19. C. H. Bennett, D. P. DiVincenzo, J. A. Smolin, W. K. Wooters, Phys. Rev. A 54, 3824 (1996).

20. Y.L. Lim, A. Beige, L.C. Kwek, www.arXiv.org/quant-ph/0408043.

21. S. D. Barrett, P. Kok, www.arXiv.org/quant-ph/0408040.

22. We acknowledge fruitful conversations with T. A. B. Kennedy, J. A. Sauer, L. You, A. Zangwill and, particularly, M. S. Chapman, and thank R. Smith and E. T. Neumann for experimental assistance. This work was supported by NASA and the Research Corporation. 
Table 1: Conditional probabilities $P(I \mid S)$ to detect the idler photon in state $I$ given detection of the signal photon in state $S$, at the point of maximum correlation for $\Delta t=100 \mathrm{~ns}$ delay between read and write pulses; all the errors are statistical.

\begin{tabular}{ccccc} 
Basis & $P\left(H_{i} \mid H_{s}\right)$ & $P\left(V_{i} \mid H_{s}\right)$ & $P\left(V_{i} \mid V_{s}\right)$ & $P\left(H_{i} \mid V_{s}\right)$ \\
\hline 0 & $0.92 \pm 0.02$ & $0.08 \pm 0.02$ & $0.88 \pm 0.03$ & $0.12 \pm 0.03$ \\
45 & $0.75 \pm 0.02$ & $0.25 \pm 0.02$ & $0.81 \pm 0.02$ & $0.19 \pm 0.02$
\end{tabular}




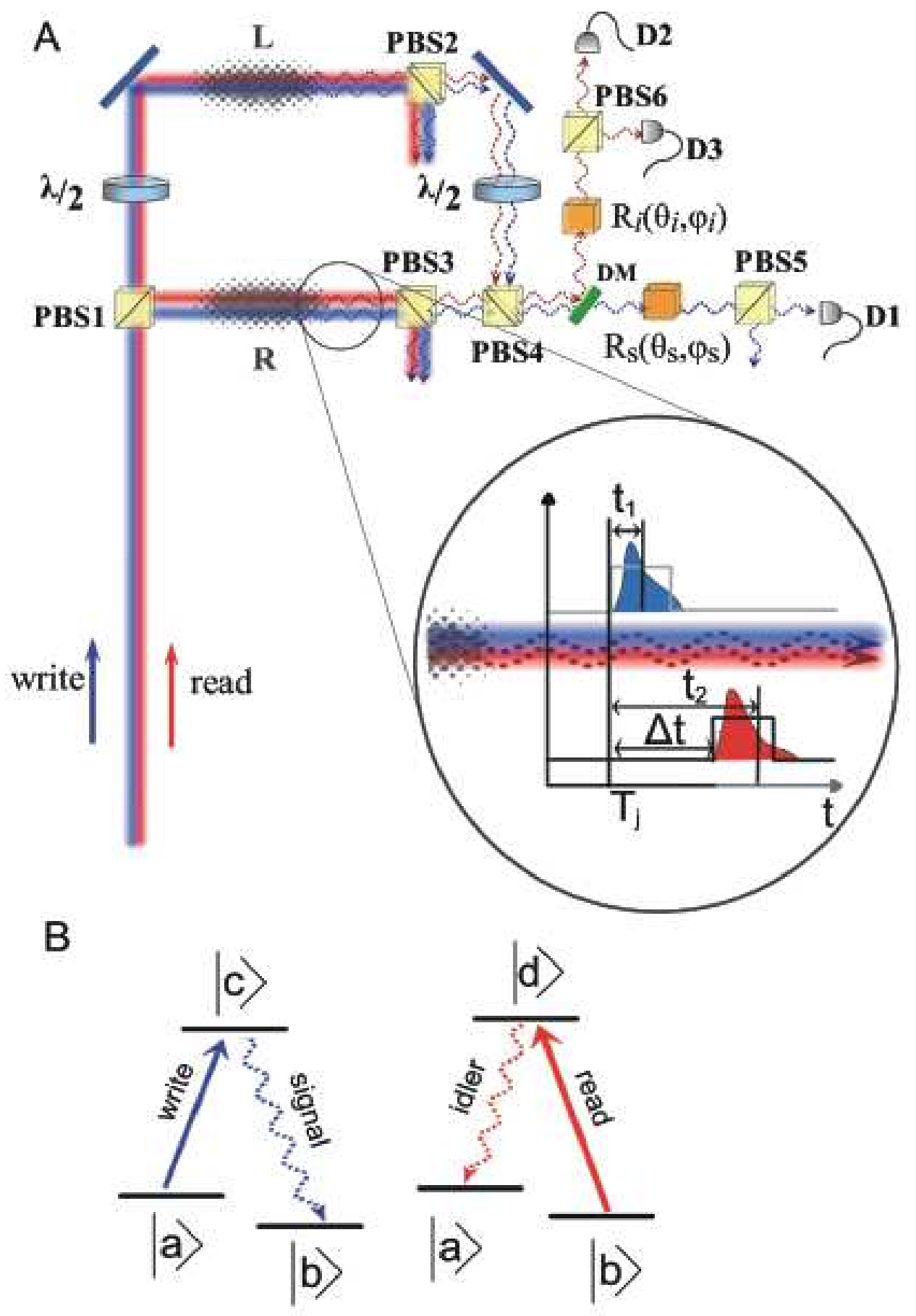

Figure 1: (A) Schematic of experimental setup. PBS1-6, polarizing beam splitters, $\lambda / 2$, half waveplate, polarization state transformers, $R_{s}\left(\phi_{1}, \phi_{s}\right)$ and $R_{i}\left(\theta_{i}, \phi_{i}\right)$, (D1,D2,D3), single photon detectors, DM, dichroic mirror. The inset illustrates the timing of the write and read pulses. (B) The relevant atomic level structure. 
A

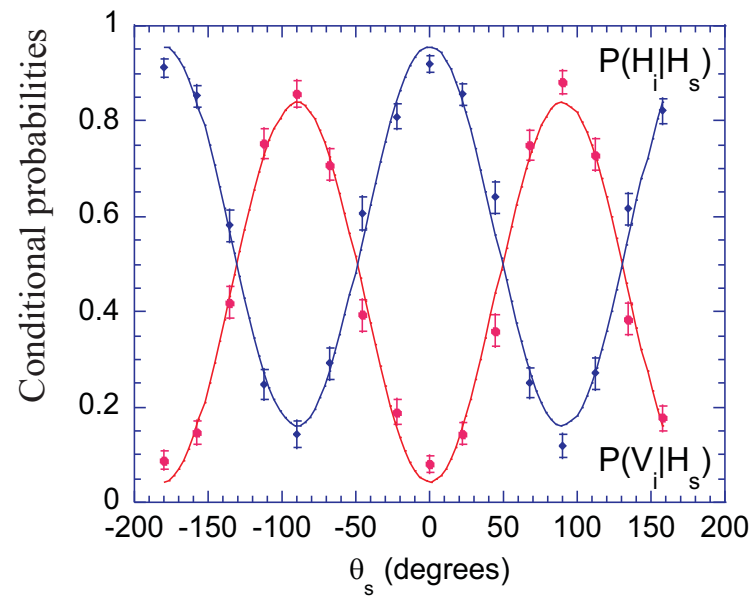

$\mathrm{B}$

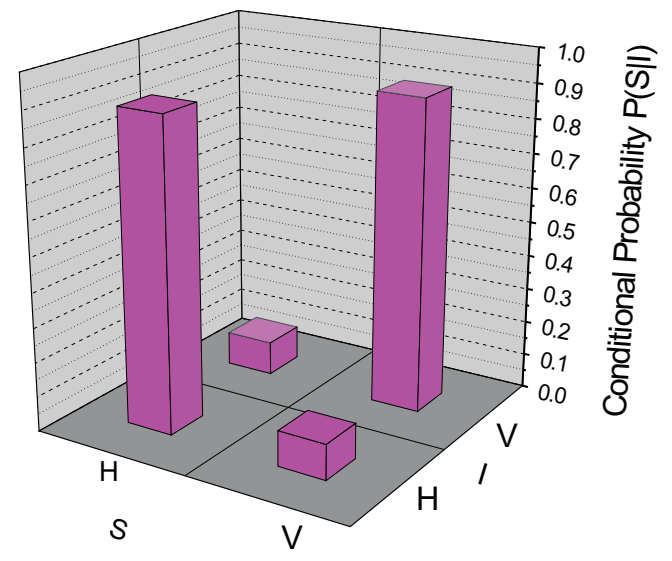

Figure 2: (A) Measured conditional probabilities $P\left(H_{i} \mid H_{s}\right)$ and $P\left(V_{i} \mid H_{s}\right)$ as the function of the polarization rotation $\theta_{s}$ of the signal photon. The full curves are fits with the visibility as the only adjustable parameter. (B) Measured conditional probabilities at the points of highest correlation. 
A

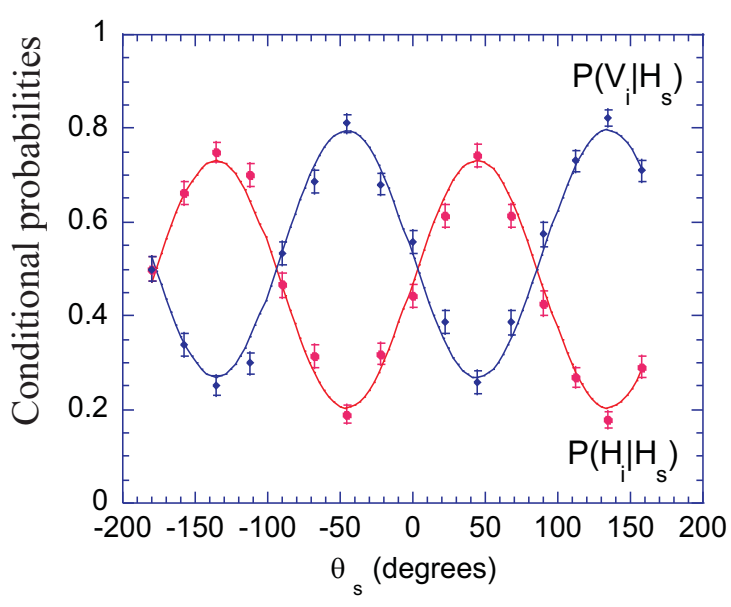

B

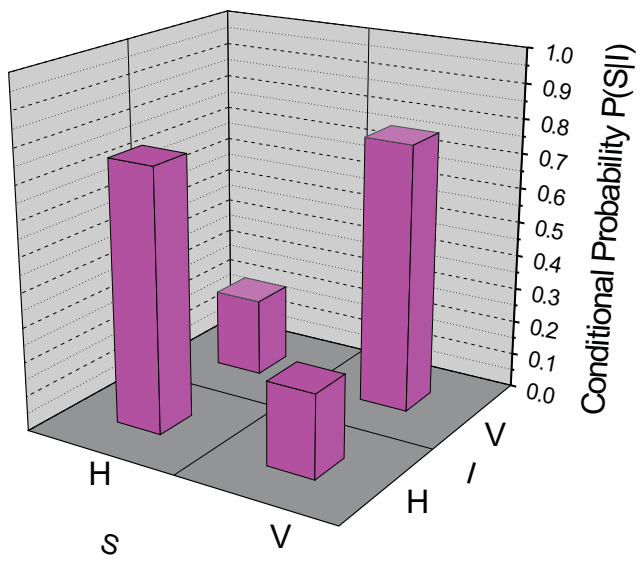

Figure 3: (A) Measured conditional probabilities after $\theta_{i}=\pi / 4$ polarization rotation of the idler photon as the function of $\theta_{s}$. (B) Measured conditional probabilities at the points of highest correlation. 


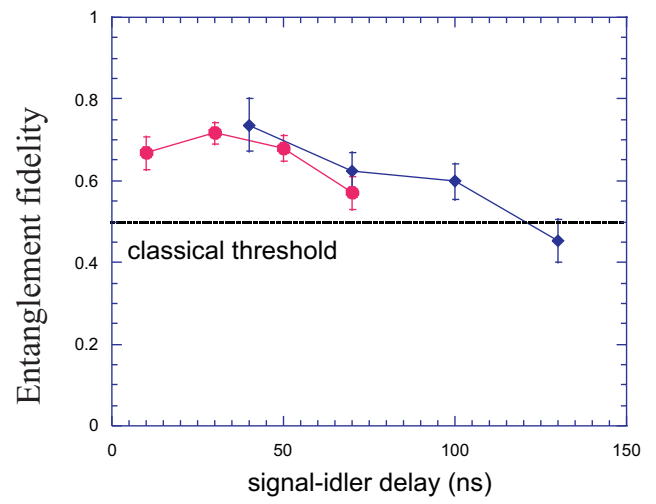

Figure 4: Time-dependent entanglement fidelity of the signal and the idler $F_{s i}$; circles for $\Delta t=$ $100 \mathrm{~ns}$, diamonds for $\Delta t=200 \mathrm{~ns}$. 